\title{
Confocal Ellipsoidal Reflector System for a Mechanically Scanned Active Terahertz Imager
}

\author{
Nuria Llombart, Member, IEEE, Ken B. Cooper, Member, IEEE, Robert J. Dengler, Member, IEEE,
} Tomas Bryllert, and Peter H. Siegel, Fellow, IEEE

\begin{abstract}
We present the design of a reflector system that can rapidly scan and refocus a terahertz beam for high-resolution standoff imaging applications. The proposed optical system utilizes a confocal Gregorian geometry with a small mechanical rotating mirror and an axial displacement of the feed. For operation at submillimeter wavelengths and standoff ranges of many meters, the imaging targets are electrically very close to the antenna aperture. Therefore the main reflector surface must be an ellipse, instead of a parabola, in order to achieve the best imaging performance. Here we demonstrate how a simple design equivalence can be used to generalize the design of a Gregorian reflector system based on a paraboloidal main reflector to one with an ellipsoidal main reflector. The system parameters are determined by minimizing the optical path length error, and the results are validated with numerical simulations from the commercial antenna software package GRASP. The system is able to scan the beam over $0.5 \mathrm{~m}$ in cross-range at a $25 \mathrm{~m}$ standoff range with less than $1 \%$ increase of the half-power beam-width.
\end{abstract}

Index Terms-Reflector antennas, scanning antennas, submillimeter-wavelength imaging, terahertz radar, THz.

\section{INTRODUCTION}

$\mathbf{R}$ ECENTLY attention has focused on defense and security terahertz (THz) applications because signals at these frequencies can penetrate many garments and provide moderate resolution images of the body at long standoff ranges without any exposure to ionizing radiation. One promising approach

Manuscript received July 01, 2009; revised November 16, 2009; accepted December 25, 2009. Date of publication March 29, 2010; date of current version June 03,2010 . This work was carried out by the Jet Propulsion Laboratory, California Institute of Technology, under a contract with the National Aeronautics and Space Administration. This work was supported under a contract to the California Institute of Technology, Division of Biology, by the Naval Explosive Ordnance Disposal Technology Division, with funding provided by the DoD Physical Security Equipment Action Group (PSEAG).

N. Llombart was with the Jet Propulsion Laboratory, California Institute of Technology, Pasadena, CA 91109 USA. She is now with the Optics Department, Universidad Complutense de Madrid, E-28040 Madrid, Spain (e-mail: nuria. llombart@opt.ucm.es).

K. B. Cooper and R. J. Dengler are with the Jet Propulsion Laboratory, California Institute of Technology, Pasadena, CA 91109 USA.

T. Bryllert was with the Jet Propulsion Laboratory, California Institute of Technology, Pasadena, CA 91109 USA. He is now with the Physical Electronics Laboratory, Chalmers University of Technology, SE-412 96 Gothenburg, Sweden and also with Wasa Millimeter Wave AB, 42341 Torslanda, Sweden.

P. H. Siegel is with the Jet Propulsion Laboratory, California Institute of Technology, Pasadena, CA 91109 and also with the Department of Biology, California Institute of Technology, Pasadena, CA 91125 USA (e-mail: phs@ caltech.edu).

Color versions of one or more of the figures in this paper are available online at http://ieeexplore.ieee.org.

Digital Object Identifier 10.1109/TAP.2010.2046860 to $\mathrm{THz}$ imaging utilizes the frequency-modulated continuouswave (FMCW) radar technique, where at submillimeter wavelengths, ultra-high resolution range measurements are used to detect objects concealed under clothing. In the FMCW radar imager described in [1], a 570-600 GHz radar beam is focused onto targets at $4 \mathrm{~m}$ standoff range using a folded-path antenna system with a $40 \mathrm{~cm}$ diameter ellipsoidal main reflector. To obtain imagery with a single transceiver located at the reflector's close focus, the entire radar platform was mechanically rotated about two axes at rates of a few degrees per second. This slow scanning speed limited the THz radar's imaging rate to roughly 10-20 ms per pixel, or one useful frame every several minutes. Other THz imaging systems have implemented advanced scanning mechanism to steer the main beam, as for example in [2], where two off-axis continuously rotating reflectors are used achieve a fast vertical scan without any acceleration, and in [3], where a periscope-based conical scan is implemented. However these systems rely on steering the beam after the main aperture, making them difficult to be used with large diameter apertures.

A goal of the next-generation $\mathrm{THz}$ imaging radar system is to greatly increase the imaging speed [4] as well as the standoff range distance. The system will be operating at $25 \mathrm{~m}$ with a $1 \mathrm{~m}$ antenna aperture. The cross-range field of view of the system was chosen to be $0.5 \times 0.5 \mathrm{~m}$ in area to span a field of view the size of a human torso. One route to achieving fast imaging is to fabricate a camera-like imaging system, where a focal plane radar array would acquire information from several pixels simultaneously. However, this approach would require a very large development cost because $\mathrm{THz}$ heterodyne detector array technology is in its infancy [5], [6]. Therefore we consider here an alternative that while still relying on mechanical scanning of a single beam projected onto a target, can nonetheless achieve rapid imaging by rotating a small, lightweight secondary mirror to steer the beam. We estimate that the imaging radar's frame rate could increase by up to two orders of magnitude in this way [4]. Moreover, our design leaves open the possibility of zooming the $\mathrm{THz}$ beam's focal point throughout a swath of near field distances in order to attain high quality imagery of targets over a long span of standoff ranges. The idea of using a small rotating mirror in order to increase the imaging acquisition time has already been used in several existing active $\mathrm{THz}$ imagers [7]-[9]. In [7] and [8], a telecentric lens design is used to focus each target pixel into a collimated beam. The collimated beams can be steered with a small mirror towards the frontand back-end electronics. In [9] a large focusing mirror is used in combination with a small rotation mirror that will steer the beam towards different parts of the mirror before focusing to 
the target. These optical designs are very effective in terms of acquisition time. However, they pay a large cost in resolution because the main apertures are very under illuminated.

The scanning THz imager's design proposed here uses a nearfield or confocal Gregorian reflector system (CGRS) [10]-[13] consisting of two paraboloid reflectors sharing a common focus. This type of reflector system has been proposed for satellite applications because of its excellent scanning performance [13], [14], a consequence of a cancellation of the coma and astigmatism aberrations that are normally present in more conventional reflector configurations [15]. In that proposed satellite application of CGRS, a phased array is used to perform the beam scanning, while for the terrestrial $\mathrm{THz}$ imaging of persons addressed here, we propose using a small rotating mirror for beam steering due to the technical challenge of scaling phased array technology to submillimeter wavelengths. In [16], beam scanning was proposed to be achieved by using small rotating reflectors in the aperture-image space of two reflectors. A tertiary reflector was shaped in order to have very good beam quality while scanning by rotational movement of this reflector. In our design, the rotating mirror is illuminated by a collimated beam rather than a expanding beam as in [16]. This is important because it relaxes the tolerances on the position of the rotating mirror's principal axes, which otherwise would be difficult to align at these high frequencies.

The paper is divided as follows. Section II presents the geometry of the reflector system. Section III studies the distortions of the scanned beam over the symmetric plane of a CGRS, while Section IV extends the design to a system that focuses in the near-field using an ellipsoidal reflector. In Section V we consider the reflector's ability to refocus at variable ranges using a displacement of the feed. Finally, Section VI discusses the possibility of simultaneous scanning in the azimuth and elevation directions using the secondary mirror.

\section{REFLECTOR ANTENNA GeOMETRY}

We consider first a CGRS based on a paraboloidal main reflector and subreflector, with diameters $D_{m}$ and $D_{s}$, as shown in Fig. 1. These parabolas share the same focal point, which is taken as the origin of the system. The focal distances of both paraboloidal reflectors are related by the system magnification as $M=F_{m} / F_{s}$. The overall dimension of the antenna system will depend on this magnification and the f-number $\left(F_{m} / D_{m}\right)$ of the system. The main reflector is offset by $X_{0}$ in the XZ plane. The beam is steered in the YZ plane, $\theta_{s b}$, by rotating an angle $\theta_{s}$ about the $\mathrm{x}$-axis the flat mirror (diameter $D_{r}$ ) located at a distance $L_{r}$ from the subreflector. The flat mirror is illuminated by a paraboloidal feed reflector, diameter $D_{f}$, in order to achieve plane wave incidence over the secondary reflector. The focal distance and angle of the feed reflector, $F_{f}$ and $\theta_{f}$, can be adjusted to optimize the far field characteristics of the available feeds and the refocusing requirements.

The THz imager described in [1] uses a silicon etalon beam splitter to duplex the transmit and receive signals. In the antenna geometry of Fig. 1, the signal duplexing can be done at the feed reflector level by placing the beam splitter between the horn and the feed reflector. The characteristics of the feed reflector can also be changed to accommodate this beam splitter without (a)

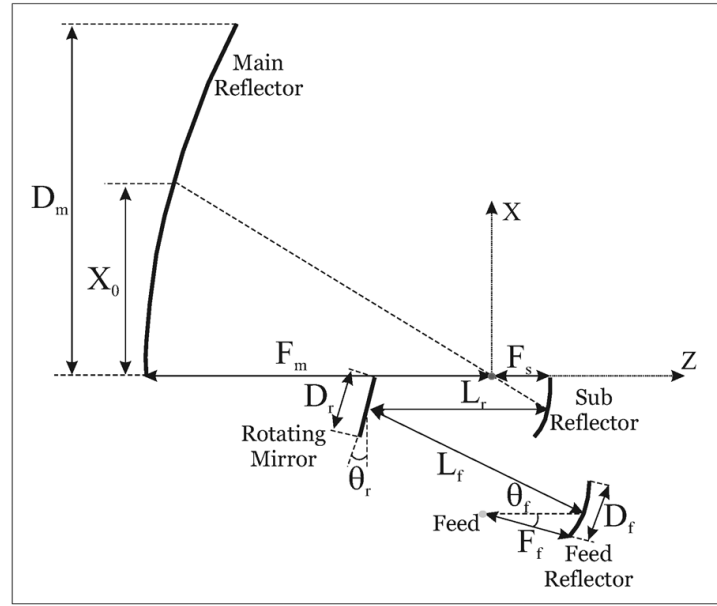

(b)

(c)

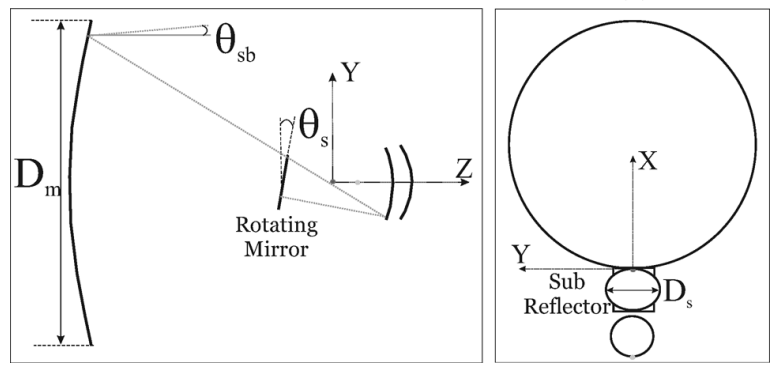

Fig. 1. Paraboloid CGRS geometry: (a) XZ-, (b) YZ- and (c) XY-planes.

affecting the scanning performance. Another more compact way of duplexing is to use a waveguide coupler.

\section{Study of the ScAnNing Performance}

For $\mathrm{cm}$-scale 3D radar imaging of targets at standoff ranges of $25 \mathrm{~m}$, diffraction-limited resolution requires the main aperture to have a diameter of about $1 \mathrm{~m}$. The typical cross-range span needed for imagery of persons is $0.5 \mathrm{~m}$ ( or $\theta_{s b}= \pm 0.57^{\circ}$ ), which means that the necessary angular displacement of the rotating mirror can be determined by the beam deviation factor to be: $\theta_{s}=M \theta_{s b} B D F / 2$, where $B D F$ is the beam deviation factor [17], and it is defined as the ratio between the main beam scan angle and the subreflector scan angle. The $B D F$ varies between 0.7 and 1 depending on the system $\mathrm{f}$-number and offset. If the reflector is uniformly illuminated, this corresponds to scanning by approximately \pm 20 half-power beam-widths (HPBW) at $670 \mathrm{GHz}$.

The first order distortions associated with beam scanning can be assessed by computing the path length error, referred as $\epsilon$ in this paper, over the main aperture [15]. This error is the difference between the length of each ray and the length of the central one. As explained in [13], the CGRS has superior scanning performances because the coma and astigmatism errors associated with asymmetric path length errors cancel, leaving a quadratic path length error for the confocal geometry.

Fig. 2 shows the ray picture for a plane wave incident at $\theta_{s b}=0.6^{\circ}$ on a symmetric confocal system with $M=4$ and $F_{m}=1.5 \mathrm{~m}$. The rays are only computed up to the rotating flat mirror for a $2 \mathrm{D}$ cut. The corresponding path length 


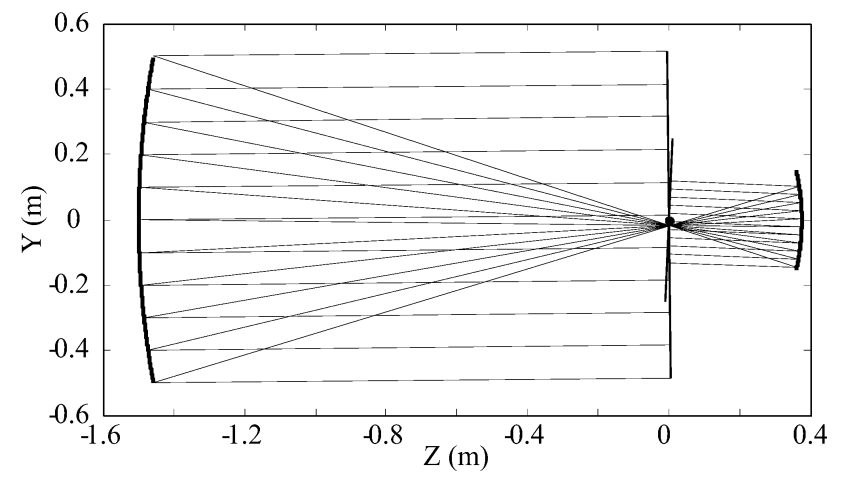

Fig. 2. Picture of the ray tracing of a CGRS system with $F_{m}=1.5 \mathrm{~m}, M=4$, $D_{m}=1 \mathrm{~m}$ and $X_{0}=0$ for a plane wave incident at $\theta_{s b}=0.6^{\circ}$.

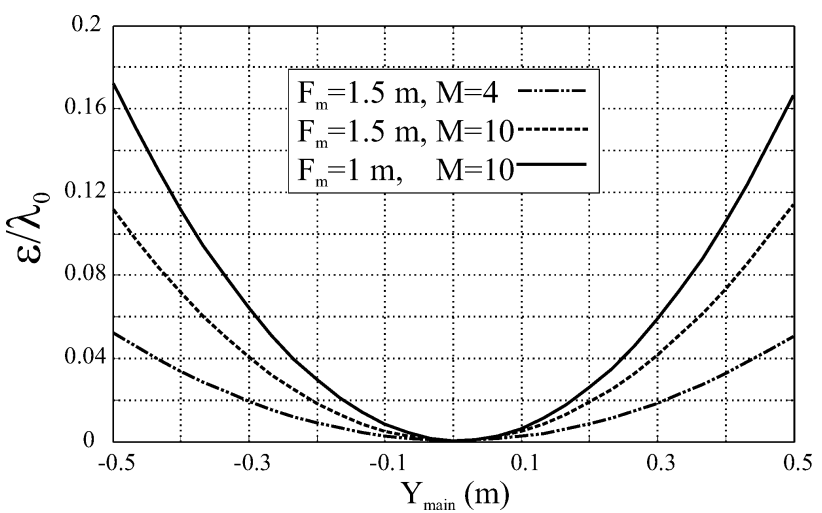

Fig. 3. Normalized path length error of a symmetric Paraboloid CGRS considering different system parameters as a function of the main aperture dimension for a plane wave incident at $\theta_{s b}=0.6^{\circ}$.

error is shown in Fig. 3. In this case the size of the rotating mirror is $D_{r} \approx 30 \mathrm{~cm}$, which is probably too large for significant imaging speed improvement. At a larger magnification $M=10$, the rotating mirror would be correspondingly smaller in size $\left(D_{r} \approx 12 \mathrm{~cm}\right)$, but at a cost of a larger path length error (Fig. 3). A second tradeoff for larger magnification is that the rotational angle $\theta_{s}$ needed for a fixed beam scanning distance scales with $M$, potentially presenting a greater challenge to the mechanical implementation of a fast rotation [4].

We have considered an f-number of 1.5 up to now, but this implies a quite large system since $D_{m}=1 \mathrm{~m}$. Therefore smaller f-numbers are preferable, but Fig. 3 shows that the path length error for an f-number of 1 is substantially worse for the $M=$ 10 case. We believe that a good compromise between system size and distortion is achieved when $F_{m}=1.2 \mathrm{~m}$ and $M=$ 10, and in Fig. 4 we summarize the results of a physical optics simulation of the entire antenna system using GRASP for these parameters.

The system feed for this geometry was chosen to be the Picket-Potter horn [18], which is commonly used at submillimeter wavelengths. This feed is modeled as a Gaussian beam with a taper of $10 \mathrm{~dB}$ at $12^{\circ}$. Fig. $4 \mathrm{a}$ and $\mathrm{b}$ show the far field for the center and scanned $\left(\theta_{s}=3^{\circ}\right)$ beams, respectively. The insets of the figures show the corresponding far field beam intensities with respect to $u=\sin \theta \cos \phi$ and $v=\sin \theta \sin \phi$. When the mirror is in the central position, we obtain a pattern with a HPBW of $0.0305^{\circ}$ and a spillover loss of $0.39 \mathrm{~dB}$. (a)

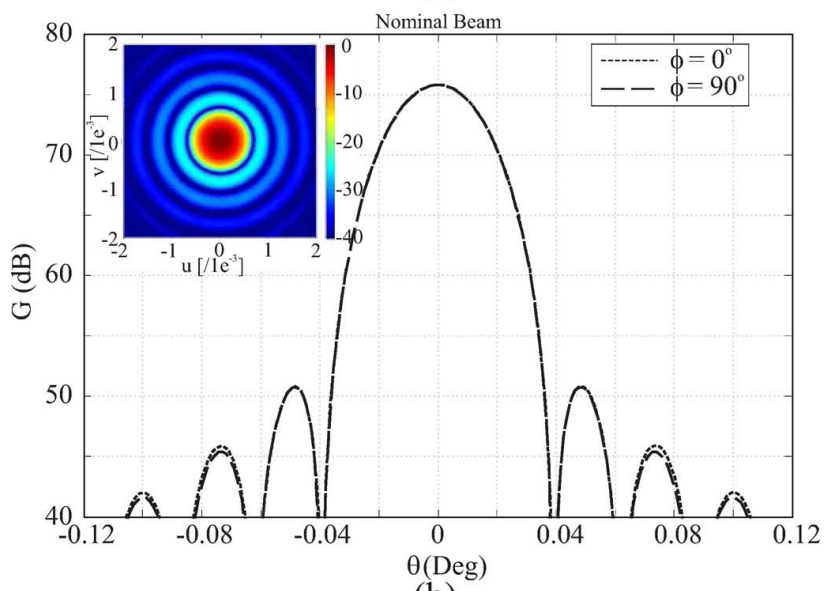

(b)

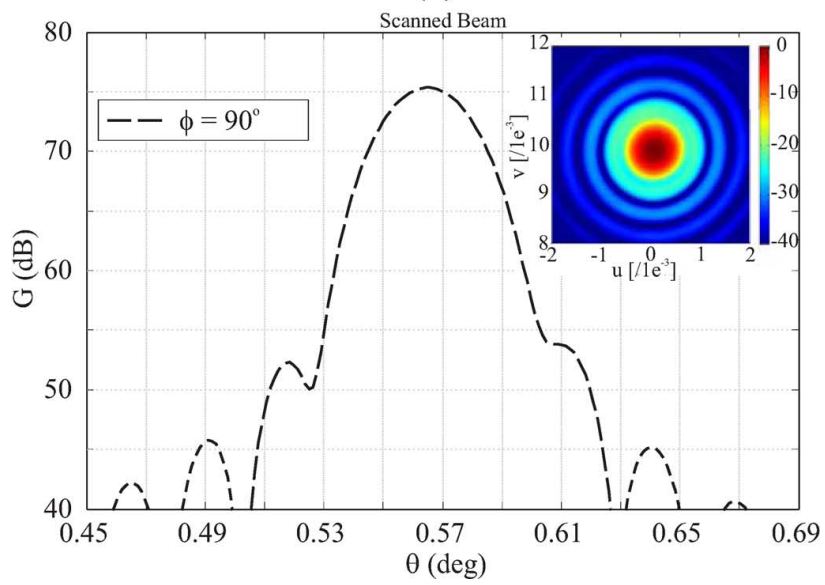

Fig. 4. Paraboloid CGRS: Far field for $\theta_{s}=0^{\circ}$ (a) and $3^{\circ}$ (b), where $\phi=0^{\circ}$ refers to the offset plane (XZ) and $\phi=90^{\circ}$ to the symmetrical plane (YZ). The inset of the figures shows the far field $u v$-grids. The main geometrical parameters are $D_{m}=1 \mathrm{~m}, M=10, F_{m}=1.2 \mathrm{~m}, X_{0}=0.55 \mathrm{~m}, L_{r}=0.19 \mathrm{~m}$, $\theta_{r}=20^{\circ}, L_{f}=0.31 \mathrm{~m}, F_{s}=0.22 \mathrm{~m}$ and $\theta_{f}=14.25^{\circ}$.

With the flat mirror fully rotated to $\theta_{s}=3^{\circ}$, the HPBW is hardly affected at all, only increasing by $1 \%$. In both cases, the cross-polarization fields fall outside the shown scale.

Optimization of the secondary reflector's shape, for example by using a bifocal structure [19] or by deforming the sub-reflector surface [13], might result in even less beam distortion. However, the fields shown in Fig. 4 are more than adequate for imaging purposes, with virtually no change in the crossrange resolution (i.e., the HPBW) over the $\pm 25 \mathrm{~cm}$ beam scan. GRASP simulations indicate that even larger scan angles are possible with minimal distortion; for a scanning distance of $\pm 37 \mathrm{~cm}$ the HPBW only increases by $8 \%$.

\section{NEAR FIELD FoCUSING}

The previous section considered the confocal Gregorian system with a paraboloidal main reflector, which provides focusing in the far field. However, at a $25 \mathrm{~m}$ standoff distance, targets are electrically very close to the antenna (in the reactive near field) for a $670 \mathrm{GHz}$ radar. This means the main reflector must be an ellipsoid to achieve diffraction-limited focusing at $25 \mathrm{~m}$. To a first order approximation, the near field patterns of 


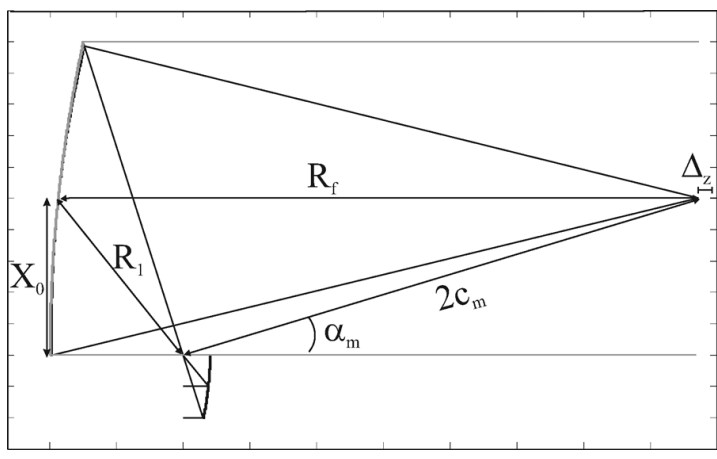

Fig. 5. Geometry of a CGRS with an ellipsoidal reflector as main aperture.

an ellipsoid will be the same as the far field patterns of a paraboloid, as presented in the previous section, as long as the main rays of the two mirror types are coincident and the ellipse's eccentricity is not too large. The geometrical mapping of the paraboloidal main reflector to the corresponding ellipsoidal reflector is summarized in Fig. 5. The mapping consists of having the same values of $R_{1}$ and $X_{0}$ for both types of reflectors, as well as having the seconday focus of the ellipsoidal reflector at $X_{0}$ and the desired focusing distance $R_{f}$ from the reflector. The ellipsoidal surface is then defined by the major axis distance $2 a_{m}=R_{1}+R_{f}$, the foci distance $2 c_{m}$, and the axis tilt angle $\alpha_{m}$. This approximate optical equivalence of paraboloidal and ellipsoidal reflectors was used by the authors for the fist time in [24] where a compensated Gregorian system with reduced cross-polarization was designed. Its primary utility is that existing design rules for far field systems (for example, the low cross-polarization dual-reflector system [21] and general design rules [22], [23]) can be readily applied to the near field.

We have thus adapted the antenna geometry developed for Figs. 1-4 to an ellipsoidal main reflector, and Fig. 6 shows the resulting ray picture for a CGRS with $M=10$ and $R_{f}=25 \mathrm{~m}$. The inset shows a magnified view of the rays around the main reflector region. For near-field focusing, the beam is scanned towards $\Delta_{s} \approx R_{f} \theta_{s b}$; see Fig. 6 . The path length error calculation for this system, where the length of the rays is computed starting from the rotating mirror up to the second focal plane (see Fig. 6), is shown in Fig. 7 as a function of the rotating mirror's $y$-coordinate, which relates to the $y$-coordinate of the main reflector as $Y_{\text {main }} \approx M Y_{\text {mirror }}$. The normalized error amplitude is seen to be less than 0.35 over the entire span, which is comparable to the results of Fig. 3. The small distortions that do arise are attributed to the system actually focusing to a slightly different distance with respect to the nominal when scanning. Fig. 7 shows that the error becomes much smaller if the second focal plane is displaced by a distance $\Delta_{z}=0.35 \mathrm{~m}$ (defined in Fig. 5), before increasing again at $\Delta_{z}=0.5 \mathrm{~m}$. A larger f-number system will have the minimum plane of distortion closer to the actual focusing distance, i.e., $\Delta_{z}$ is smaller. For the imaging application considered here, this focusing plane displacement is not very significant.

In order to check the paraboloidal/ellipsoidal design equivalence, the ellipsoidal CGRS was simulated with GRASP, and the field values at $25 \mathrm{~m}$ are presented in Fig. 8. As expected from the

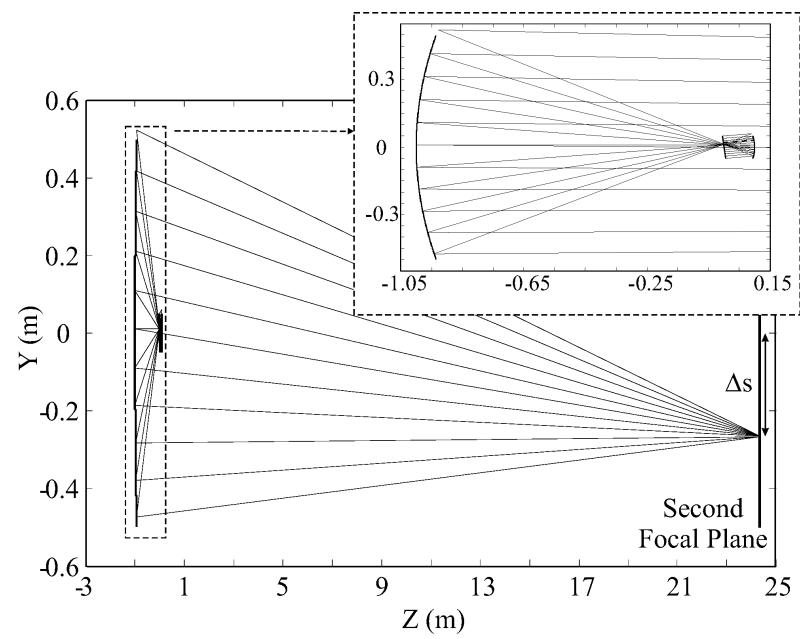

Fig. 6. Ray picture for an ellipsoidal CGRS with $F_{m}=1 \mathrm{~m}, M=10$, $D_{m}=1 \mathrm{~m}, R_{f}=25 \mathrm{~m}$ and $\theta_{s}=-3^{\circ}$.

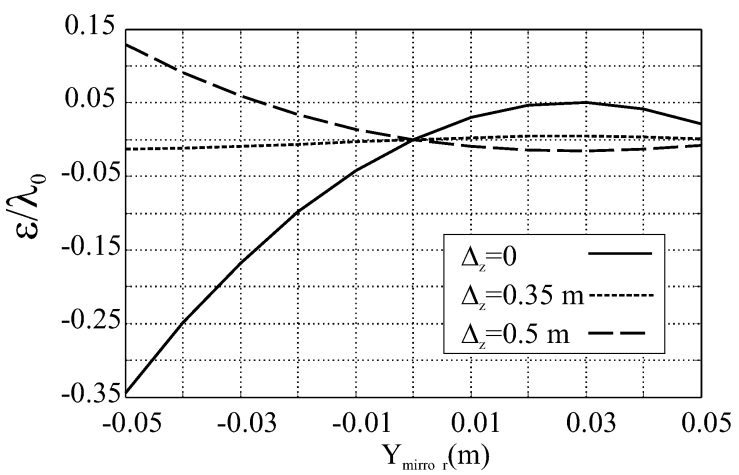

Fig. 7. Path length error for a symmetric CGRS with an ellipsoidal main reflector, and $F_{m}=1 \mathrm{~m}$ and $M=10$ as a function of the second focal plane distance for $\theta_{s}=3^{\circ}$.

design equivalence, the fields at $\theta_{s}=0^{\circ}$ and $3^{\circ}$ exhibit virtually the same HPBW and spillover losses as the ones associated with the paraboloidal reflector. In fact, the beam profiles would be indistinguishable from the ones shown in Fig. 4 if they were plotted on top of one another. The HPBW of the center beam at $25 \mathrm{~m}$, corresponding to $0.0305^{\circ}$, is $\Delta_{x}=\Delta_{y}=1.33 \mathrm{~cm}$.

\section{REFOCUSING THE IMAGING SYSTEM}

In this section an approach for focusing at different near-field distances is considered. In particular, we propose to achieve zooming by the mechanical displacement of a system component. As with beam steering, the alternative of phased array zooming [25], while very attractive, is prohibited by the available technology at $\mathrm{THz}$ frequencies. Our goal is to evaluate refocusing over a $\pm 50 \%$ deviation covering the span $12.5 \mathrm{~m}-$ $37.5 \mathrm{~m}$ in range.

A single ellipsoidal reflector has two optimal focuses defined by the ellipse parameters, and one can focus to a different secondary focus by axially displacing the first focal point by $\Delta_{f}$ as shown in the inset Fig. 9. This displacement results in the second focus of the ellipse moving from $R_{f}^{0}$ to $R_{f}^{\Delta}$. Fig. 9 shows the ray picture of an offset single reflector where the feed is displaced by $\Delta_{z}=14.2 \mathrm{~cm}$. This displacement will introduce a certain path length error as shown in Fig. 10. The feed displacement is 
(a)

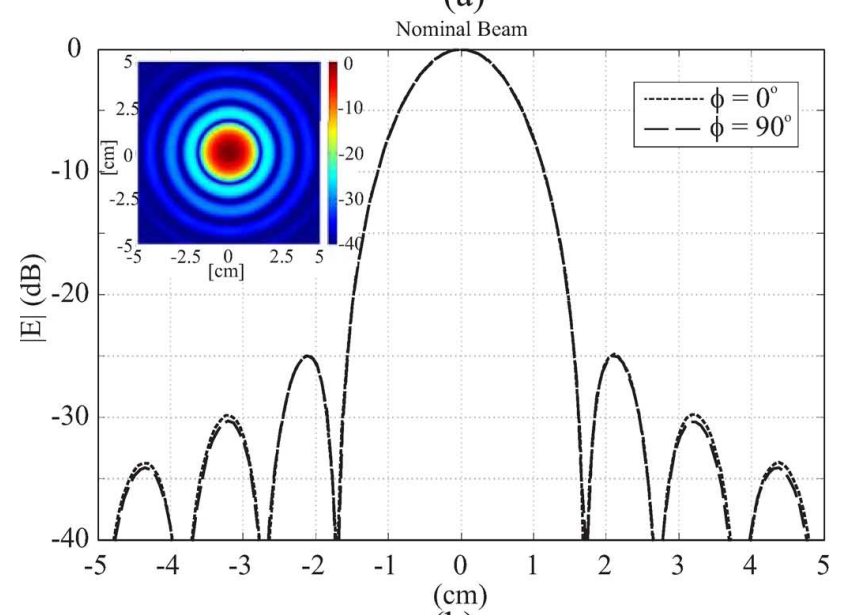

(b)

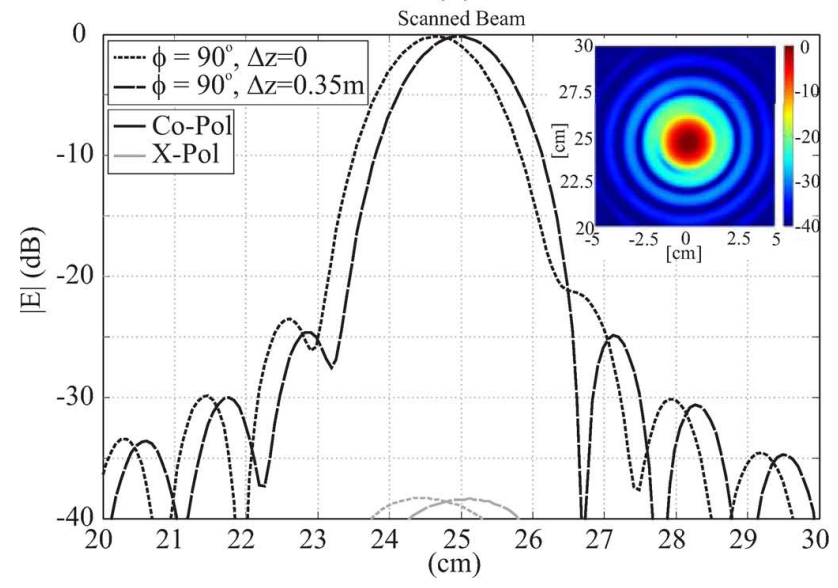

Fig. 8. Ellipsoidal CGRS: Near field at $25 \mathrm{~m}$ for $\theta_{s}=0^{\circ}$ (a) and $3^{\circ}$ (b). The inset of the figures shows the near field $x y$-grids. The system has the same geometrical parameters as Fig. 4 plus $R_{f}=25 \mathrm{~m}, \alpha_{m}=1.32^{\circ}$ and $2 c_{m}=$ $23.869 \mathrm{~m}$

chosen by minimizing the path length error computed up to $R_{f}^{\Delta}$ in each of the main planes. For offset reflectors, the optimum feed displacement is different in the symmetrical $\left(\phi=90^{\circ}\right)$ and offset planes $\left(\phi=0^{\circ}\right)$ as can be seen Fig. 10. In the case of a larger $\mathrm{f}$-number, the relative difference between the optimum distances for each plane is smaller as well as the maximum path length error, resulting in higher quality beams, as can be seen in the same figure. Fig. 11 shows the near fields at $12.5 \mathrm{~m}$ for two offset reflectors with different f-numbers. The asymmetrical path length error of Fig. 10 for $\phi=0^{\circ}$ results in significant coma and beam tilting distortions. In an actual system, the feed offset will be in between the optimum distances for each plane, and it would be a compromise between the beam qualities in both planes.

In the confocal geometry, the focal point of the main ellipsoidal reflector needs to be displaced in order to refocus the system. One could achieve the refocusing by translating the whole secondary optical system (i.e., secondary reflector, rotating mirror, feed reflector and feed). In such a case the beam quality will depend on the main reflector's f-number, which has been fixed to 1.2 in previous sections in order to keep the system

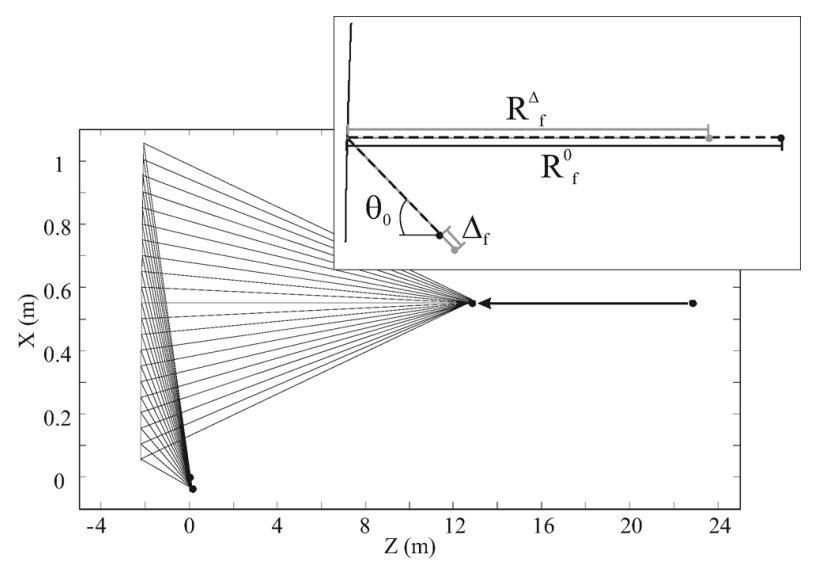

Fig. 9. Ray picture for an ellipsoidal reflector with $f=2.2, X_{0}=0.55 \mathrm{~m}$, $R_{f}^{0}=25 \mathrm{~m}, R_{f}^{\Delta}=15 \mathrm{~m}$ and $\Delta_{z}=14.2 \mathrm{~cm}$. The inset of the figure shows the geometrical description of a single ellipsoidal reflector with the feed displaced $\left(\Delta_{f}\right)$ to change the focusing distance towards $R_{f}^{\Delta}$ from the nominal one $R_{f}^{0}$.

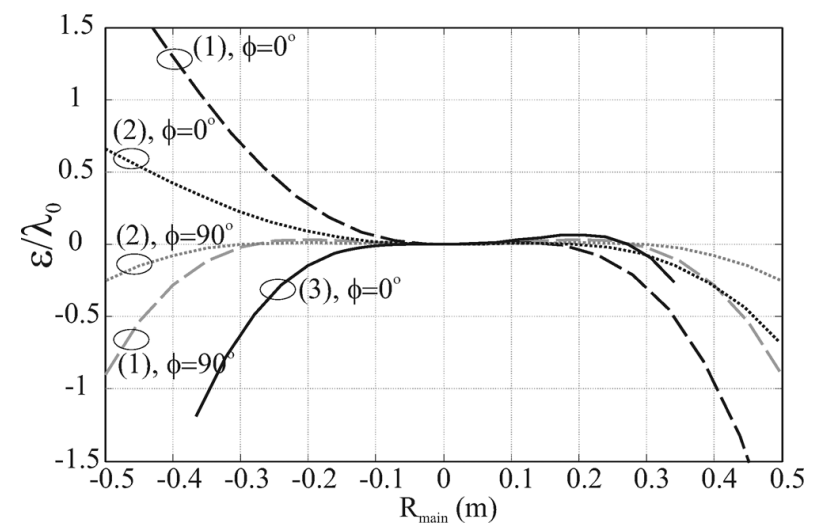

Fig. 10. Path length error when refocusing at $12.5 \mathrm{~m}$ for several reflector system configurations: (1) Single reflector, SR, $\left(F_{m}=1.2 \mathrm{~m}\right.$ and $\left.X_{0}=0.55 \mathrm{~m}\right)$ where $\Delta_{f}=6.3 \mathrm{~cm}$ for $\phi=0^{\circ}$ and $\Delta_{f}=7.3 \mathrm{~cm}$ for $\phi=90^{\circ}$; (2) SR, $\left(F_{m}=2.2 \mathrm{~m}\right.$ and $\left.X_{0}=0.55 \mathrm{~m}\right)$ where $\Delta_{f}=21.5 \mathrm{~cm}$ for $\phi=0^{\circ}$ and $\Delta_{f}=22.65 \mathrm{~cm}$ for $\left.\phi=90^{\circ}\right)$; and (3) same CGRS of Fig. $8\left(\Delta_{f}=13.5 \mathrm{~cm}\right)$.

overall dimension reasonably small. This small f-number will cause large beam distortions, as shown in Fig. 11. A solution that presents better beam quality is the displacement of the feed only. The advantage here is that the f-number of the feed reflector is higher (i.e., 2.2). The phase error associated with the confocal geometry is also shown in Fig. 10 when only the feed is displaced. The feed displacement provided in the figure caption has been chosen as a compromise between the beam qualities in both planes, $\phi=0^{\circ}$ and $90^{\circ}$. This error is comparable to that of the single reflector with the same f-number. However the main reflector is under illuminated (see Figs. 10 and 12). This aperture illumination depends on the distances $L_{r}$ and $L_{f}$, which have been chosen to avoid blockage effects when displacing the feed for refocusing at $25 \mathrm{~m} \pm 12.5 \mathrm{~m}$. Because of this poorer illumination, the HPBW associated with the confocal geometry is larger than the one of the single reflector. However, the beam tilting effect and side lobe level in the offset cut $(\phi=0)$ are much better for the CRGS, as indicated by the smaller beam length error. Fig. 13 shows that fields, when refocusing at 37.5 $\mathrm{m}$, have a comparable side lobe level and beam tilting effect than 
(a)

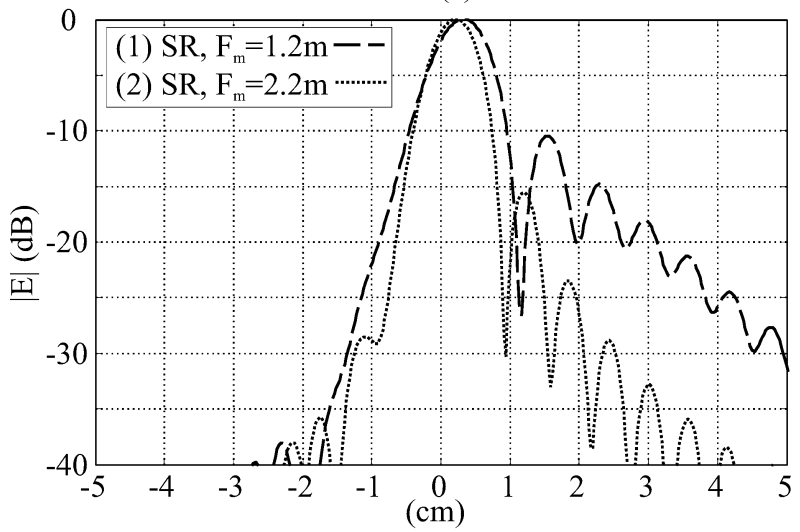

(b)

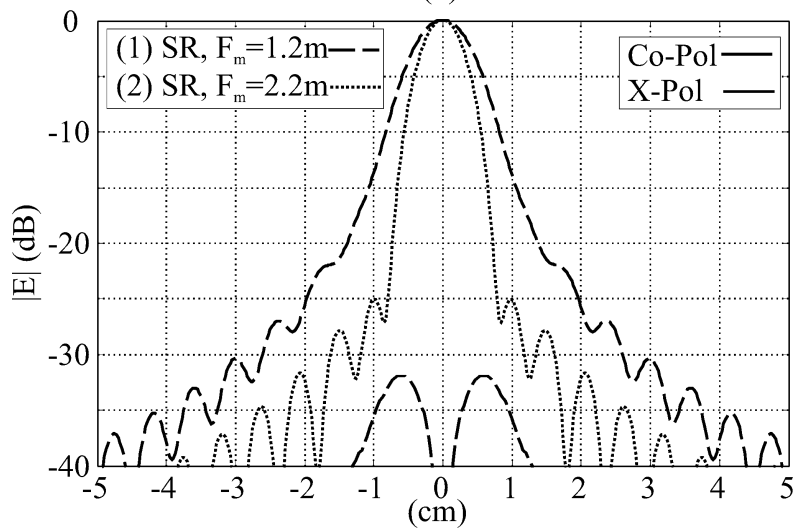

Fig. 11. Near field at $R_{f}^{\Delta}=12.5 \mathrm{~m}$ of the geometries (1)-(2) described in Fig. 10 with $\Delta_{f}=6.5 \mathrm{~cm}$ and $\Delta_{f}=22 \mathrm{~cm}$, respectively: (a) $\phi=0^{\circ}$ and (b) $90^{\circ}$ cuts.

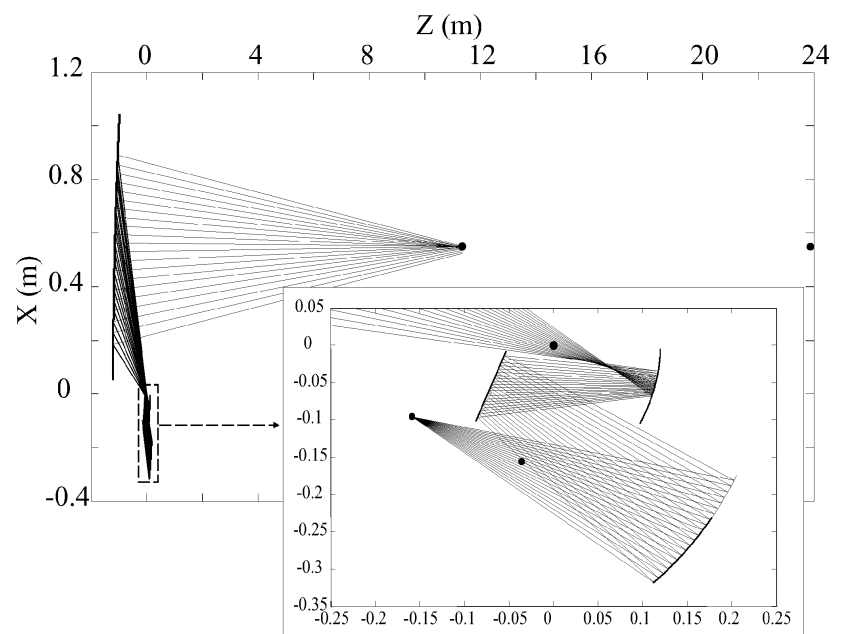

Fig. 12. Ray picture for the ellipsoidal CGRS with $\Delta_{f}=13.5 \mathrm{~cm}$. Notice that the main reflector is under illuminated.

the ones at $12.5 \mathrm{~m}$. In both cases, the cross-polarization fields fall outside the shown scale.

Finally, both operations, scanning and refocusing, can be simultaneously combined. To check the quality of the fields, we have computed the near fields at the external boundaries of the focusing region at the maximum scan angle $\left(\theta_{s}=3 \mathrm{deg}\right)$ with GRASP. The fields are shown in Fig. 14 and Table I presents a summary of the simulated parameters of the system for both

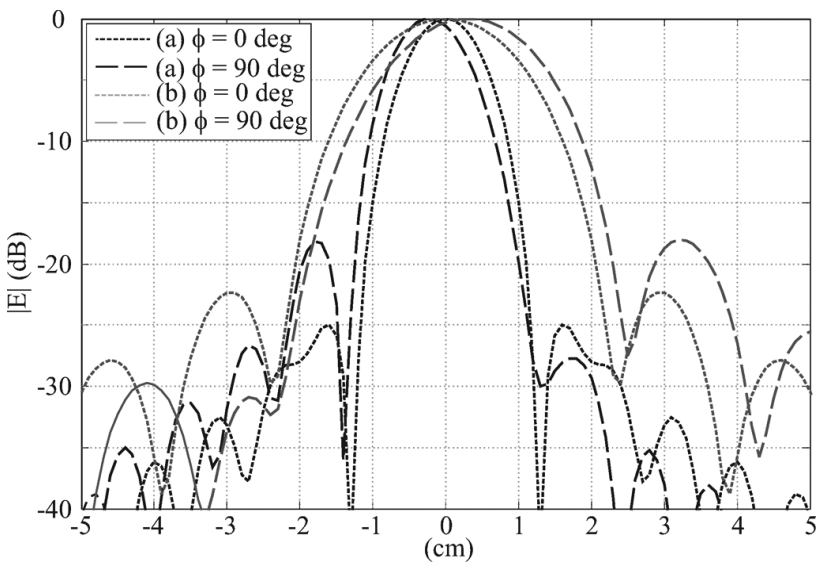

Fig. 13. Near fields at (a) $R_{f}^{\Delta}=12.5 \mathrm{~m}\left(\Delta_{f}=13.5 \mathrm{~cm}\right)$ and (b) $R_{f}^{\Delta}=$ $37.5 \mathrm{~m}\left(\Delta_{f}=-8 \mathrm{~cm}\right)$ of the ellipsoidal CGRS.

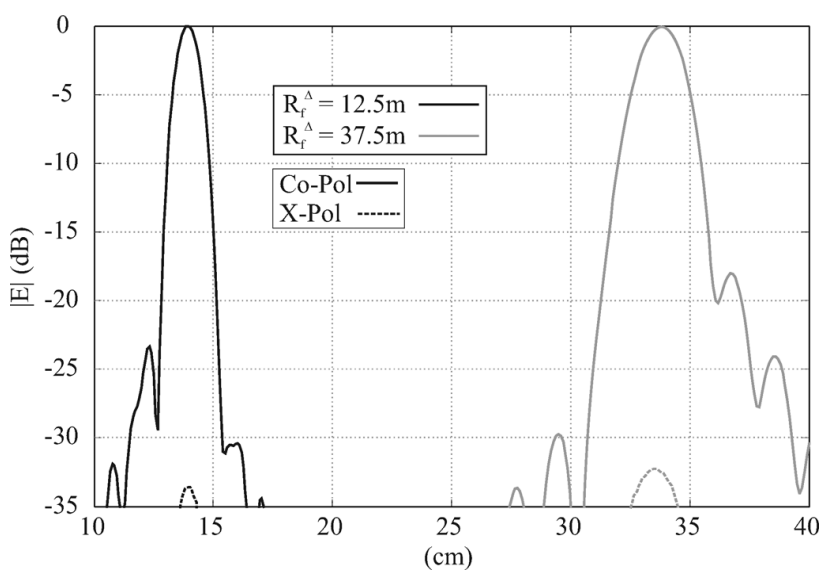

Fig. 14. Near field at $R_{f}^{\Delta}=12.5 \mathrm{~m}$ and $R_{f}^{\Delta}=37.5 \mathrm{~m}$ of the ellipsoidal CPGRS with $\theta_{s}=3^{\circ}$.

operations. Note that the spill over (SO) provided in the table is associated with a Gaussian beam excitation, and the SO associated with the actual feed pattern may be larger. Even when refocusing, the scanned beams are of excellent quality. On one hand, the spillover loss is below $0.8 \mathrm{~dB}$ over the whole scanning and focusing operation range. For example, the HPBWs $\left(\Delta_{x}\right.$ and $\Delta_{y}$ ) when scanning increase by less than $6 \%$ with respect to the center beam $\left(\theta_{s}=0^{\circ}\right)$. From Table I, we can see that $\Delta_{x}$ and $\Delta_{y}$ at the several focusing distances are different from each other. Actually, if the main aperture would have the same illumination when refocusing, i.e., the same spillover loss, the HPBW at $12.5 \mathrm{~m}$ and $37.5 \mathrm{~m}$ would be half and one and a half that at $25 \mathrm{~m}$, respectively. Instead, the actual values are larger and smaller than these ideal ones because of different illumination of the main reflector. Even so, they are within a factor of two of one another and should provide comparable imaging performance at the different distances.

\section{2-AXIS SCANNING SYSTEM}

A flat secondary mirror that can rotate in two planes could achieve faster frame rates than a single-axis mirror by steering the radar beam in a circular or spiral pattern [26]. Therefore it is valuable to examine the beam quality in the orthogonal scanning plane (the XZ plane of Fig. 1 for the antenna configurations 
TABLE I

SCANNING AND REFOCUSING PARAMETERS OF THE ELLIPSOIDAL CGRS

\begin{tabular}{|c|c||c|c|c|c|c|}
\hline $\begin{array}{c}\Delta_{f} \\
{[\mathrm{~cm}]}\end{array}$ & $\begin{array}{c}\theta_{s} \\
{\left[{ }^{o}\right]}\end{array}$ & $\begin{array}{c}R_{f}^{\Delta}[\mathrm{m}] \\
{\left[\begin{array}{c}{[\mathrm{cm}]}\end{array}\right.}\end{array}$ & $\begin{array}{c}\Delta_{x} \\
{[\mathrm{~cm}]}\end{array}$ & $\begin{array}{c}\mathrm{SO} \\
{[\mathrm{dB}]}\end{array}$ & $\begin{array}{c}\Delta_{s} \\
{[\mathrm{~cm}]}\end{array}$ \\
\hline \hline 0 & 0 & 25 & 1.33 & 1.33 & 0.39 & 0 \\
\hline 0 & \pm 3 & 25 & 1.34 & 1.33 & 0.40 & \pm 24.62 \\
\hline 13.5 & 0 & 12.5 & 0.99 & 0.99 & 0.33 & 0 \\
\hline 13.5 & \pm 3 & 12.5 & 1.04 & 1.02 & 0.33 & \pm 13.95 \\
\hline-8 & 0 & 37.5 & 1.87 & 1.87 & 0.70 & 0 \\
\hline-8 & \pm 3 & 37.5 & 1.97 & 1.96 & 0.76 & \pm 33.84 \\
\hline
\end{tabular}

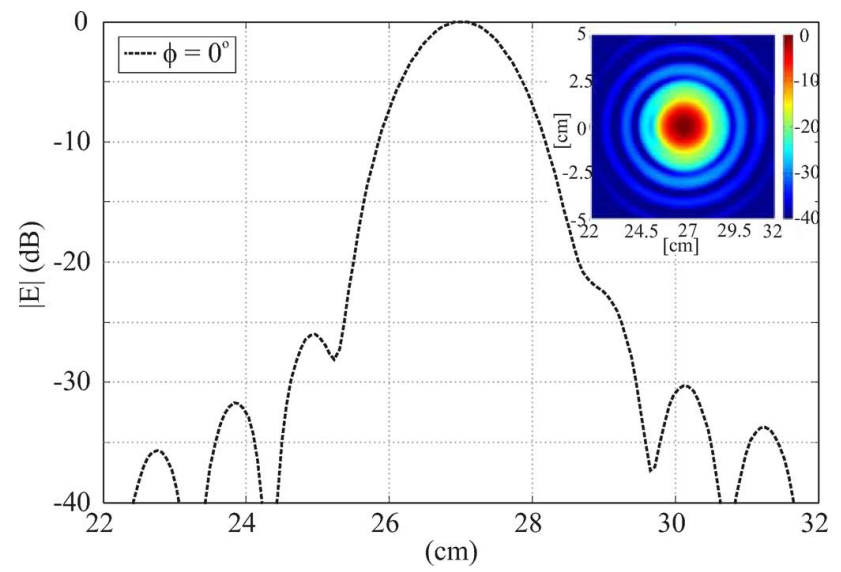

Fig. 15. Near field at $25 \mathrm{~m}$ when scanning in the offset plane $\left(\theta_{s}=-3^{\circ}\right.$, $\phi_{s}=0^{\circ}$ ).

TABLE II

PARAMETERS OF THE 2D SCANNING ELLIPSOIDAL CGRS

\begin{tabular}{|c|c||c|c|c|c|}
\hline $\begin{array}{c}\theta_{s} \\
\left.{ }^{o}\right]\end{array}$ & $\begin{array}{c}\phi_{s} \\
{\left[{ }^{\circ}\right]}\end{array}$ & $\begin{array}{c}\Delta_{x} \\
{[\mathrm{~cm}]}\end{array}$ & $\begin{array}{c}\Delta_{y} \\
{[\mathrm{~cm}]}\end{array}$ & $\begin{array}{c}\mathrm{SO} \\
{[\mathrm{dB}]}\end{array}$ & $\begin{array}{c}\Delta_{s} \\
{[\mathrm{~cm}]}\end{array}$ \\
\hline \hline+3 & 0 & 1.3 & 1.31 & 0.39 & -25.5 \\
\hline-3 & 0 & 1.34 & 1.33 & 0.27 & 27 \\
\hline \pm 3 & 90 & 1.31 & 1.31 & 0.32 & \pm 23.8 \\
\hline
\end{tabular}

presented here). In order to avoid beam blockage effects, simulations were performed with a larger offset, $X_{0}$, of $60 \mathrm{~cm}$ rather than $55 \mathrm{~cm}$ used in Fig. 8. Fig. 15 shows the scanned beam when the flat mirror is rotated by $-3^{\circ}$ in this offset plane $\left(\phi_{s}=0^{\circ}\right)$. As shown in Table II, which compares the beam widths and spillover loss for scanning in the two directions, there will be only a negligible impact (at most 3\%) on the radar's imaging resolution when the beam is steered in any direction up to $\pm 3^{\circ}$. The asymmetry of the HPBWs when scanning towards positive or negative angles for $\phi=0^{\circ}$ is associated with the offset of the structure.

\section{CONCLUSION}

The next generation of terahertz imagers will require fast scanning and will benefit from refocusing capabilities. In this paper, a reflector system design was presented that can achieve both functionalities using mechanical rotation and translation of small secondary optical elements while the large primary mirror remains stationary. The reflector geometry consists on a confocal system. Using this system, very low path length errors are obtained when either scanning or refocusing the beam, or both operations are applied, yielding to very low beam distortions. The design was implemented by generalizing a confocal dual reflector system with a paraboloidal main reflector into a near-field focusing system with an ellipsoidal main reflector using a simple equivalence rule. The beam patterns were numerically calculated using GRASP for all extreme system specifications of scan angle $\left( \pm 3^{\circ}\right)$ and refocusing distance (50\% from the nominal). These results indicate that the $\mathrm{THz}$ imaging radar system will able to scan a target area of $0.5 \mathrm{~m}$ at $25 \mathrm{~m}$ stand-off with less than $3 \%$ increase of the HPBW at the nominal focusing distance, and to refocus from $12.5 \mathrm{~m}$ up to $37.5 \mathrm{~m}$ while maintaining $\mathrm{cm}$-scale imaging resolution.

\section{REFERENCES}

[1] K. B. Cooper, R. J. Dengler, N. Llombart, T. Bryllert, G. Chattopadhyay, E. Schlecht, J. Gill, C. Lee, A. Skalare, I. Mehdi, and P. H. Siegel, "Penetrating 3D imaging at 4 and 25 meter range using a submillimeter-wave radar," IEEE Trans. Microw. Theory Tech., vol. 56, pp. 2771-2778, Dec. 2008.

[2] A. H. Lettington, D. Dunn, N. E. Alexander, A. Wabby, B. N. Lyons, R. Doyle, J. Walshe, M. F. Attia, and I. Blankson, "Design and development of a high-performance passive millimeter-wave imager for aeronautical applications," SPIE Opt. Eng., vol. 44, p. 093202, 2005.

[3] E. N. Grossman, C. R. Dietlein, M. Leivo, A. Rautiainen, and A. Luukanen, "A passive, real-time, terahertz camera for security screening, using superconducting microbolometers," in Proc. IEEE Int. Microwave Symp., Jun. 7-12, 2009, pp. 1453-1456.

[4] K. B. Cooper, R. J. Dengler, N. Llombart, T. Bryllert, G. Chattopadhyay, I. Mehdi, and P. H. Siegel, "An approach for sub-second imaging of concealed weapons using terahertz (THz) radar," Int. J. Infrared Millim. Wave, vol. 30, no. 12, pp. 1297-1307, Dec. 2009.

[5] C. Groppi, C. Walker, C. Kulesa, D. Golish, J. Kloosterman, P. Pütz, S. Weinre, T. Kuiper, J. Kooi, G. Jones, J. Bardin, H. Mani, A. Lichtenberger, T. Cecil, A. Hedden, and G. Narayanan, "SuperCam: A 64 pixel heterodyne imaging spectrometer," in Proc. SPIE, 2008, vol. 7020, pp. 702011-1-8.

[6] G. Chattopadhyay, "Heterodyne arrays at submillimeter wavelengths," presented at the XXVIIIth General Assembly of Int. Union of Radio Science, New Delhi, India, Oct. 2005.

[7] T. M. Goyette, J. C. Dickinson, K. J. Linden, W. R. Neal, C. S. Joseph, W. J. Gorveatt, J. Waldman, R. Giles, and W. E. Nixon, "1.56 terahertz 2 -frames per second standoff imaging," in Proc. SPIE, Terahertz Technology and Applications, Jan. 2008, vol. 6893, Photonics West 2008.

[8] J. Xu and G. C. Cho, "A real-time terahertz wave imager," presented at the Lasers and Electro-Optic and Conf. on Quantum Electronics and Laser Science Conf. CLEO/QELS, May 4-9, 2008.

[9] Q. Song, A. Redo-Sanchez, Y. Zhao, and C. Zhang, "High speed imaging with CW THz for security," in Proc. SPIE, 2008, vol. 7160, pp. 716016-716016-8.

[10] C. Dragone and M. J. Gans, "Imaging reflector arrangements to form a scanning beam using a small array," Bell Syst.Tech. J., vol. 58, no. 2 , pp. 501-515, Feb. 1979.

[11] S. Morgan, "Some examples of generalized Cassegrain and Gregorian antennas," IEEE Trans. Antennas Propag., vol. 12, no. 6, pp. 685-691, Nov. 1964.

[12] W. D. Fitzgerald, Limited Electronic Scanning with an Offset-Feed Near-Field Gregorian System MIT Lincoln Lab., 1971, Tech. Rep. 486.

[13] J. A. Martinez-Lorenzo, A. Garcia-Pino, B. Gonzalez-Valdes, and C. M. Rappaport, "Zooming and scanning Gregorian confocal dual reflector antennas," IEEE Trans. Antennas Propag., vol. 56, no. 9, pp. 2910-2919, Sep. 2008.

[14] Y. Imaizumi, Y. Suzuki, Y. Kawakami, and K. Araki, "A study on an onboard Ka-band phased-array-fed imaging reflector antenna," in Proc. IEEE Antennas and Propag. Society Int. Symp., 2002, vol. 4, pp. $144-147$.

[15] C. Dragone, "A first-order treatment of aberrations in Cassegrain and Gregorian antennas," IEEE Trans. Antennas Propag., vol. AP-30, no. 3, pp. 331-339, 1982.

[16] P. C. Werntz, W. L. Stutzman, and K. Takamizawa, "A high-gain trireflector antenna configuration for beam scanning," IEEE Trans. Antennas Propag., vol. 42, no. 9, pp. 1205-1214, Sep. 1994. 
[17] A. W. Rudge, K. Milne, A. Olver, and P. Knight, The Handbook of Antenna Design. London, U.K.: Peter Peregrinus.

[18] P. D. Potter, "A new horn antenna with suppressed sidelobes and equal beamwidths," Microw. J., p. 71, Jun. 1963.

[19] J. Rao, "Bicollimated Gregorian reflector antenna," IEEE Trans. Antennas Propag., vol. 32, no. 2, pp. 147-154, Feb. 1984.

[20] F. Ulaby, R. Moore, and A. Fung, Microwave Remote Sensing. Norwood, MA: Artech House, 1982.

[21] Y. Mizugutch, M. Akagawa, and H. Yokoi, "Offset dual reflector antennas," in Proc. IEEE Antennas and Propag. Society Int. Symp., Oct. 1976, pp. 2-5.

[22] K. W. Brown and A. Prata, "A design procedure for classical offset dual reflector antennas with circular apertures," IEEE Trans. Antennas Propag., vol. 42, no. 8, Aug. 1994.

[23] W. V. T. Rusch, A. Prata, Y. Rahmat-Samii, and R. A. Shore, "Derivation and application of equivalent paraboloid for classical offset Cassegrain and Gregorian antennas," IEEE Trans. Antennas Propag., vol. 38 , no. 8, pp. 1141-1149, Aug. 1990

[24] N. Llombart, A. Skalare, and P. H. Siegel, "High-efficiency array for submillimeter-wave imaging applications," presented at the IEEE Antennas and Propag. Soc. Inter. Symp., Jul. 5-11, 2008.

[25] W. H. Carter, "On rrefocusing a radio telescope to image sources in the near field of the antenna array," IEEE Trans. Antennas Propag., vol. 37, no. 3, pp. 314-319, Mar. 1989.

[26] C. am Weg, W. von Spiegel, B. Hills, T. Loeffler, R. Henneberger, R. Zimmermann, and H. G. Roskos, "Fast active THz camera with range detection by frequency modulation," Int. J. Infrared Millim. Wave, vol. 30, no. 12, pp. 1281-1296, Dec. 2009.

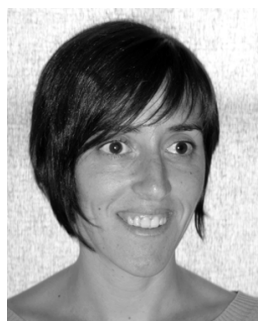

Nuria Llombart (S'06-M'07) received the Ingeniero de Telecomunicación degree and the Ph.D. from the Universidad Politécnica de Valencia, Spain, in 2002 and 2006 respectively.

During her Master's degree studies she spent one year at the Friedrich-Alexander University of Erlangen-Nuremberg, Germany, and worked at the Fraunhofer Institute for Integrated Circuits, Erlangen, Germany. From 2002 until 2007, she was with the Antenna Unit at the TNO Defence, Security and Safety Institute, The Hague, The Netherlands, working as Ph.D. student and afterwards as Researcher. From 2007 until 2009, she was a Postdoctoral Fellow at the California Institute of Technology, Pasadena, working for the SWAT group of the Jet Propulsion Laboratory. Since January 2010, she is a "Ramón y Cajal" researcher at the Universidad Complutense de Madrid, Spain. Her research interests include the analysis and design of printed array antennas, EBG structures, reflector antennas, lens antennas and submillimeter-wave components.

Dr. Llombart was a co-recipient of the 2008 H. A. Wheeler Applications Prize Paper Award from the IEEE Antennas and Propagation Society.

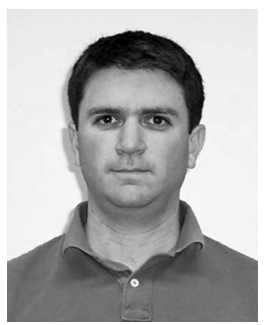

Ken B. Cooper (M'06) received the A.B. degree in physics (summa cum laude) from Harvard College, Cambridge, MA, in 1997, and the Ph.D. degree in physics from the California Institute of Technology, Pasadena, in 2003.

Since 2006, he has been a Member of the Technical Staff with the Jet Propulsion Laboratory, Pasadena, CA. His current research interests include submillimeter-wave radar, spectroscopy, and device physics.

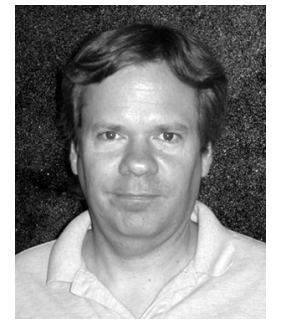

Robert J. Dengler (M'09) received the B.Sc. degree in electrical and computer engineering from California State Polytechnic University, Pomona, in March 1989.

He began his work with Dr. Siegel at the Jet Propulsion Laboratory as an intern in 1988, developing beam pattern acquisition and analysis software. Since then he has been involved in the design and construction of submillimeter-wave receivers and components, including design and fabrication of test instrumentation for submillimeter flight mixers. His recent work is focused on $\mathrm{THz}$ active imaging and heterodyne spectrometers, including design and construction of a $110 \mathrm{~dB}$ dynamic range biosample transmission imaging system at $2.5 \mathrm{THz}$, a high resolution imaging radar system operating at $670 \mathrm{GHz}$, and an ultra high-sensitivity room-temperature 550-620 GHz absorption spectrometer.

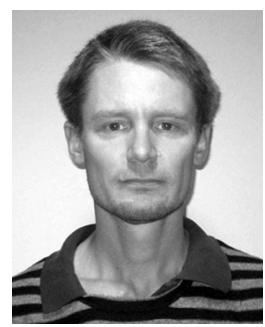

Tomas Bryllert received the degree of M.S. degree in physics and the Ph.D. degree in semiconductor physics from Lund University, Sweden, in 2000 and 2005, respectively.

In 2006, he joined the Microwave Electronics Laboratory, Chalmers University of Technology, Sweden, where his main research interest was device- and circuit-technology for terahertz frequency multipliers. During 2007-2009 he was with the Jet Propulsion Laboratory (JPL), Pasadena, CA, funded by a research fellowship from the Wallenberg foundation - working on submillimeter-wave imaging radar and terahertz time-domain imaging systems. Starting in September 2009, he joined the Physical Electronics Laboratory at Chalmers University of Technology, working on circuits and devices for millimeter wave applications. Since 2007, he is also the CEO of Wasa Millimeter Wave AB, Torslanda, Sweden, a company that develops and produces millimeter wave modules.

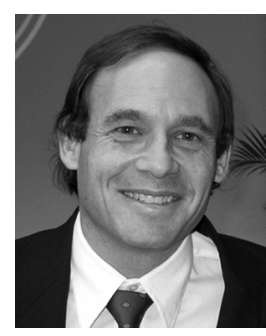

Peter H. Siegel (F'01) received the BA degree in Astronomy from Colgate University, Hamilton, NY, in 1976, and the MS in physics and Ph.D. degree in electrical engineering from Columbia University, New York, in 1978 and 1983, respectively.

He served as an NRC Fellow at the Goddard Institute for Space Studies, NY and then as a staff member in the Electronics Development Lab, National Radio Astronomy Observatory, Charlottesville, VA, until 1987. He then moved to the Jet Propulsion Laboratory, Pasadena, CA, to work on submillimeter wave sensors for NASA space astrophysics and Earth remote sensing applications. At JPL he has been involved in four space flight missions and more than 65 research and development programs. He founded and has led for more than 15 years a large technical team, SWAT - Submillimeter Wave Advanced Technology, focused on NASA applications of terahertz technology. In 2001, he joined the staff at the California Institute of Technology, where he holds appointments as Member of Professional staff in Biology and Faculty Associate in Electrical Engineering. At Caltech he has been expanding terahertz applications into biology and medicine as well as into defense and security areas. His interests cover all areas of $\mathrm{THz}$ technology, techniques and applications.

Dr. Siegel is an active member of the IEEE THz community and has served as Vice-Chair and Chair of MTT-4, THz Technology, as an IEEE Distinguished Lecturer and continuing member of the speaker's bureau, as Organizer and Chair of seven special THz sessions at sequential IMS meetings, as a long term member of the TPC and Special Guest Editor for the IEEE TRANSACTIONS ON MicrowaVe THEORY AND TEChNIQUES and JIMT. He is also Chair of the International Organizing Committee and Founding Chair of the International Society of Infrared, Millimeter, and Terahertz Waves, the largest continuous forum devoted to $\mathrm{THz}$ science and technology. 